\title{
A MAGNETOSTRICTIVE TUNING MECHANISM FOR SRF CAVITIES ${ }^{1}$
}

\author{
Chad Joshi ${ }^{2}$ and Bruce Bent, Energen, Inc., Billerica, MA
}

Michael Drury, Joseph Preble, Viet Nguyen, Jefferson Laboratory, Newport News, VA

\section{Abstract}

Energen, Inc. has designed, built and demonstrated a fine tuning mechanism for superconducting radio frequency (SRF) cavities used in particle accelerators. This tuner is based on giant magnetostrictive materials being developed by Energen, Inc. Magnetostrictors elongate when exposed to a small magnetic field. This extension is reversible and repeatable enabling a wide range of applications. The magnetostrictive tuner was specifically designed to meet the requirements of the Thomas Jefferson National Accelerator Facility in Newport News, VA. The tuner consists of a high force linear actuator that elongates the cavity along its axis thereby changing its resonant frequency. It is installed in the dead leg of the existing mechanical tuner. This mechanism has a motion range that provides a tuning range of up to $6400 \mathrm{~Hz}$. Preliminary tests at Jefferson Laboratory demonstrated cavity tuning capability.

\section{INTRODUCTION}

The Jefferson Laboratory of Newport News, VA operates the largest continuous beam electron accelerator in the world. Approximately 344 superconducting RF cavities operate at a resonant frequency of $1497 \mathrm{MHz}$ to accelerate the electrons as the they pass through each cavity. To obtain a homogeneous and continuous electron beam, these cavities are designed to exacting geometric tolerances. However, small imperfections in their manufacture and the large dimensional change that occurs as a result of cooling from room temperature to $1.8 \mathrm{~K}$ necessitates active compensation for the cavity length during the actual operation of the accelerator in order to match the resonance frequencies of multiple SRF cavities used in any accelerator.

\section{SRF CAVITY TUNING}

SRF cavity tuning is accomplished by physically elongating or compressing the cavity along its axis. Jefferson Laboratory uses a mechanical system as shown in Figure 1. The system uses a stepper motor at room temperature to drive a wormwheel gear reduction connected to a ball screw shaft. As the shaft turns, the two cell holders are squeezed together compressing the cavity and changing its resonant frequency. This mechanical system is relatively simple in concept, however, it is difficult to use for accurate cavity tuning. In operation, this system, like any other mechanical assembly, has backlash. In addition to the elastic behavior of the components, split shaft couplers are used for the rotating feedthrough to allow for thermal contraction during cooldown. This split assembly adds to the inherent backlash of the system.

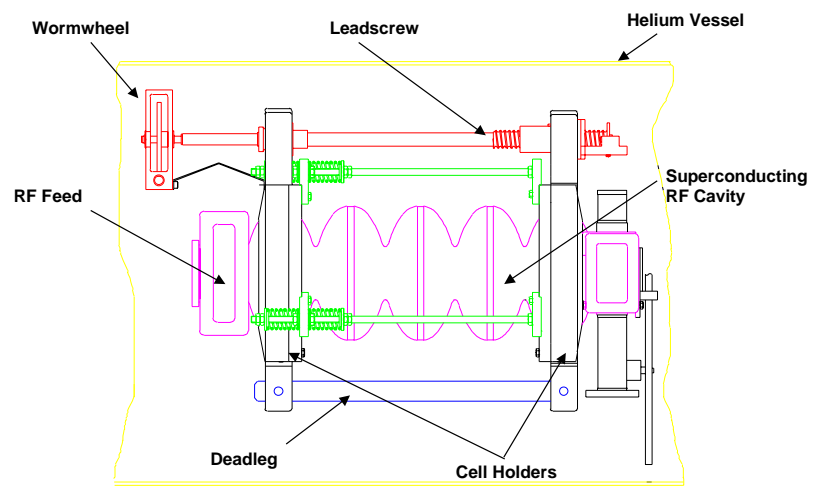

Figure 1: The CEBAF superconducting RF cavity showing its mechanical tuner.

\section{MAGNETOSTRICTIVE MATERIALS}

Magnetostrictive materials belong to a class of materials known as "smart materials". Magnetostriction arises from a reorientation of the atomic magnetic moments. In ferromagnetic materials, an applied magnetic field causes rotation of the magnetization towards the field direction within domains and/or motion of the domain walls to increase the size of the domains with magnetization vectors close in direction to the applied field. When the magnetization is completely aligned, saturation occurs and no further magnetostriction can be produced by increasing the applied magnetic field. Magnetostriction only occurs in a material at temperatures below the Curie temperature. The amount of magnetostriction at saturation is the most fundamental measure of a magnetostrictive material. Table 1 shows a compilation of some materials and the maximum strain they exhibit at saturation. Note that the materials exhibiting the highest magnetostrictive strain have Curie temperatures below room temperature. These materials show great promise for cryogenic actuator applications such as the SRF cavity tuners.

Magnetostrictive materials for cryogenic applications are not commercially available. Energen, Inc. has worked with the Ames Laboratory's Materials Preparation Center

\footnotetext{
${ }^{1}$ Supported by the Nuclear Physics Division of the U. S. Dept. of Energy through the SBIR Program

${ }^{2}$ E-mail: chad@EnergenInc.com
} 
to obtain samples of the materials for testing and building prototype devices.

Several alloys from the $T b_{1-x} D y_{x} Z n$ family of materials were fabricated as follows. Appropriate quantities of the elemental constituents were alloyed together in a sealed tantalum crucible. The crucible was then held just below the melting temperatures to promote crystal growth. Once cooled, the crucible was removed and the resulting ingot was machined into rods for testing purposes. For testing the magnetostriction, samples of each alloy were cut into $2 \mathrm{~mm}$ diameter rods of lengths varying from 20 to $30 \mathrm{~mm}$.

Table 1: Saturation strain and Curie temperatures of selected materials [1].

\begin{tabular}{l|cc}
\hline \multicolumn{1}{c|}{ Material } & $\begin{array}{c}\text { Saturation Strain } \\
\left(\mathrm{x} \mathrm{10} 0^{-6}\right)\end{array}$ & $\begin{array}{c}\text { Curie } \\
\text { Temperature } \\
(\mathrm{K})\end{array}$ \\
\hline $\mathrm{Ni}$ & -50 & 630 \\
$\mathrm{Fe}$ & -14 & 1040 \\
$\mathrm{SmFe}$ & -2340 & 690 \\
$\mathrm{Fe}_{3} \mathrm{O}_{4}$ & 60 & 860 \\
$\mathrm{DyFe}_{2}$ & 650 & 630 \\
$\mathrm{TbFe}_{2}$ & 2630 & 700 \\
$\mathrm{~Tb}_{0.3} \mathrm{Dy}_{0.7} \mathrm{Fe}_{1.9}$ (Terfenol-D) & $1600-2400$ & 650 \\
$\mathrm{~Tb}_{0.6} \mathrm{Dy}_{0.4} @ 77 \mathrm{~K}$ & 6300 & 210 \\
$\mathrm{~Tb}_{0.5} \mathrm{Zn}_{0.5}$ & 5500 & 180 \\
$\mathrm{~Tb}_{1-\mathrm{x}} \mathrm{Dy}_{\mathrm{x}} \mathrm{Zn}$ & 5000 & 200 \\
\hline
\end{tabular}

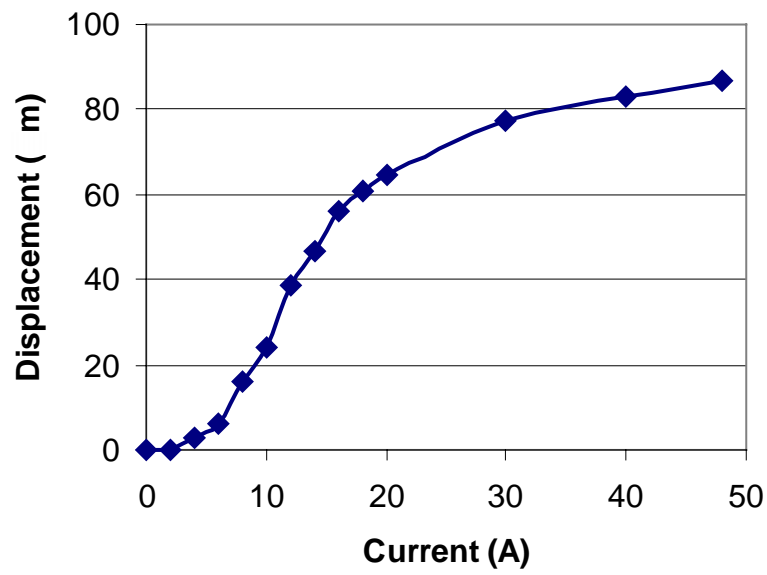

Figure 2: Magnetostriction of a TbDyZn alloy at $4.2 \mathrm{~K}$ shows high saturation strain.

Measurements of magnetostriction were made at 77 $\mathrm{K}$ and at $4.2 \mathrm{~K}$. The data for $77 \mathrm{~K}$ are very reproducible and agree with data from the literature [2]. Figure 2 shows the measurements on one sample of the TbDyZn alloy at $4.2 \mathrm{~K}$. These data are believed to be the first direct measurement of magnetostriction in this material system at $4.2 \mathrm{~K}$ and indicate that the high saturation strain remains at these low temperatures.

\section{TUNER DESIGN}

Energen, Inc. designed a magnetostrictive tuner based on these materials that replaces the deadleg of the existing mechanical tuner. This tuner was designed to provide fine tuning capability in parallel with the existing tuner. The mechanical tuner would be used for coarse tuning of the resonant frequency and then the magnetostrictive tuner would be used for fine tuning.

Figure 3 shows the magnetostrictive dead leg. Because of limitations in the size of magnetostrictor rods currently available, the magnetostrictor consists of six 0.5 inch disks of equal thickness on a 1.108" bolt circle. They are surrounded by a NbTi coil and compressed between two ferromagnetic end pieces that connect to the dead leg. The coil has 20 turns of superconductor with a critical current of $325 \mathrm{~A}$ at $4.2 \mathrm{~K}, 5.0 \mathrm{~T}$. The ferromagnetic end pieces serve to focus the magnetic flux from the coil onto the magnetostrictor. The peak field in the end pieces and in the magnetostrictor is less than 1.6 tesla.

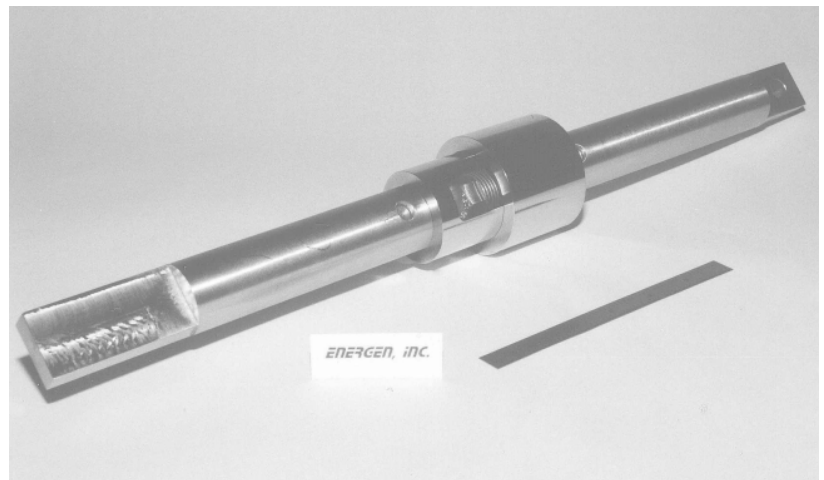

Figure 3: Magnetostrictive SRF cavity tuner designed by Energen, Inc.

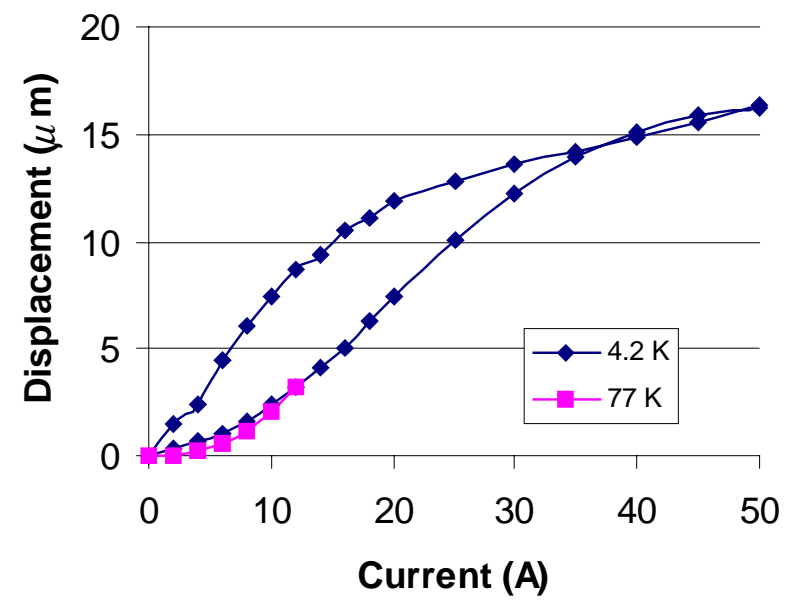

Figure 4: Measurement of dead leg tuner motion. 


\section{TUNER PERFORMANCE}

The tuner was tested at Energen's facility at $77 \mathrm{~K}$ and at $4.2 \mathrm{~K}$. At $77 \mathrm{~K}$, the current in the coil was limited to 15 A to prevent potential damage to the coil and actuator from overheating. Figure 4 shows the results of tests at $77 \mathrm{~K}$ and $4.2 \mathrm{~K}$.

Figure 4 indicates a total stroke of 16 microns is available for tuning the SRF cavity . Based on the known characteristics of the Jefferson Laboratory SRF cavities, this corresponds to a tuning bandwidth of $6400 \mathrm{~Hz}$ greater than the design goal by a factor of three.

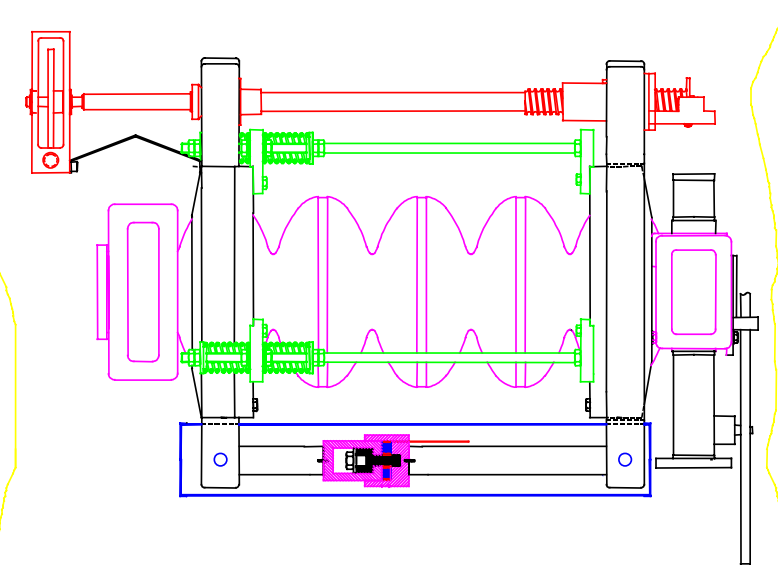

Figure 5: The magnetostrictive tuner was installed in place of the dead leg of the mechanical tuner.

It is clear that there is some hysteresis in these measurements. The source of this hysteresis is the anisotropic magnetic properties of the material and can be reduced by properly adjusting the chemistry of the material.

At Jefferson Laboratory, the tuner was installed on a SRF cavity as shown in Figure 5. In operation, a combination superconducting and mu-metal shield would be used to prevent the magnetic field from the magnetostrictive tuner from affecting the performance of the cavity.

The cavity was inserted vertically in a dewar and cooled in liquid Helium to approximately 2 Kelvin. A network analyzer (Hewlett Packard 8753) was used to drive the cavity and measure the cavity's operating frequency (fo). An RF frequency preamp was use to boost the signal from the cavity field probe before returning to the analyzer. A Sorenson programmable power supply capable of $45 \mathrm{~A}$ was used as the current source for the tuner. A 50 Amp shunt was installed in the return leg of the circuit between tuner and power supply. The shunt was calibrated for $1 \mathrm{mV} / \mathrm{A}$. A 5 1/2 digit HP multimeter was used to measure the shunt voltage. The apparatus was controlled with a laptop PC running Labview. The laptop used a GPIB card to talk to the network analyzer and the multimeter. A National Instruments analog box was attached to the parallel port. This gave an Analog output with which we could control the current source. The resolution of the current drive under this setup was about a $1 / 2 \mathrm{~A}$. The resolution of the network analyzer was about $10 \mathrm{~Hz}$.

In the first two tests, the maximum current for the tuner was $30 \mathrm{~A}$. While the pressure in the helium vessel was constant during each test, there is a slight variation over a longer period of time. This pressure affects the tuning of the cavities. During the third test, when the current was increased to $50 \mathrm{~A}$, a large change in pressure from 21 torr to 12 torr occurred during the test. There was likely a shift in frequency because of the pressure change exacerbating the hysteresis that is evident in the data.

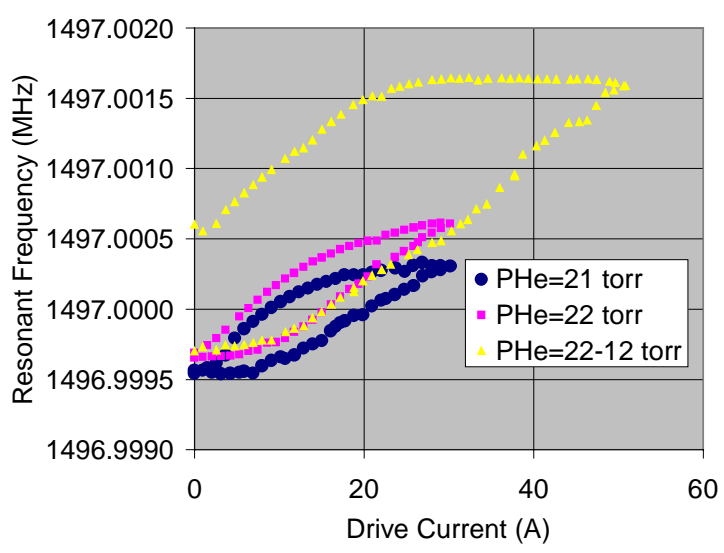

Figure 6: Tuner tests on a SRF cavity.

\section{CONCLUSION}

Energen, Inc. has designed, built and demonstrated a fine tuning mechanism for superconducting radio frequency (SRF) cavities used in particle accelerators. The tuner is designed specifically for the Jefferson Laboratory and operates in parallel with an existing mechanical tuning system that uses a wormwheel and ball screw assembly. The magnetostrictive tuner has demonstrated the desired $2000 \mathrm{~Hz}$ of tuning bandwidth and can be actively controlled during the operation of the SRF cavity. Further refinements should increase this tuning range providing an alternative tuning system to traditional mechanical tuner.

\section{REFERENCES}

[1] Magnetostrictive Materials, K. B. Hathaway and A. E. Clark, MRS Bulletin, Vol. XVIII, No. 4, April 1993.

[2] A. E. Clark, High Power Magnetostrictive Materials from Cryogenic Temperatures to $250 \mathrm{C}$, Materials Research Society Fall Meeting, Boston, MA, November 28-30, 1994. 